\title{
Cetuximab treatment for metastatic colorectal cancer with KRAS p.G13D mutations improves progression-free survival
}

\author{
HIROKI OSUMI $^{1}$, EIJI SHINOZAKI ${ }^{1}$, MASAHIKO OSAKO $^{2}$, YOSHIMASA KAWAZOE $^{3}$, MASARU OBA $^{4}$, \\ TAKAHARU MISAKA ${ }^{5}$, TAKASHI GOTO ${ }^{6}$, HITOMI KAMO ${ }^{6}$, MITSUKUNI SUENAGA ${ }^{1}$, YOSUKE KUMEKAWA ${ }^{1}$, \\ MARIKO OGURA $^{1}$, MASATO OZAKA ${ }^{1}$, SATOSHI MATSUSAKA ${ }^{1}$, KEISHO CHIN $^{1}$, \\ KIYOHIKO HATAKE $^{7}$ and NOBUYUKI MIZUNUMA ${ }^{1}$
}

\begin{abstract}
${ }^{1}$ Department of Gastroenterology, The Cancer Institute Hospital, Japanese Foundation for Cancer Research, Tokyo; ${ }^{2}$ Department of Surgery, Kagoshima Medical Association Hospital, Kagoshima, Kagoshima; ${ }^{3}$ Department of Medical Informatics and Economics, ${ }^{4}$ Division of Hepatobiliary and Pancreatic Surgery, Department of Surgery, Graduate School of Medicine, University of Tokyo, Tokyo; ${ }^{5}$ Department of Gastroenterology, Kirishima Medical Center, Kirishima, Kagoshima; ${ }^{6}$ Department of Surgery, Koga General Hospital, Miyazaki; ${ }^{7}$ Department of Medical Oncology and Hematology, New Drug Development Center, The Cancer Institute Hospital, Japanese Foundation for Cancer Research, Tokyo, Japan
\end{abstract}

Received March 13, 2015; Accepted June 11, 2015

DOI: $10.3892 /$ mco.2015.602

\begin{abstract}
A number of previous studies have reported that $30-50 \%$ of patients with colorectal cancer (CRC) harbor Kirsten rat sarcoma viral oncogene homolog (KRAS) mutations, which is a major predictive biomarker of resistance to epidermal growth factor (EGFR)-targeted therapy. Treatment with an anti-EGFR inhibitor is recommended for patients with KRAS wild-type metastatic colorectal cancer (mCRC). A recent retrospective study of cetuximab reported that patients with KRAS p.G13D mutations had better outcomes compared with those with other mutations. The aim of this retrospective study was to assess the prevalence of KRAS p.G13D mutations and evaluate the effectiveness of cetuximab in $\mathrm{mCRC}$ patients with KRAS p.G13D or other KRAS mutations. We reviewed the clinical records of $98 \mathrm{mCRC}$ patients with KRAS mutations who were treated between August, 2004 and January, 2011 in four hospitals located in Tokyo and Kyushu Island. We also investigated KRAS mutation subtypes and patient characteristics. In the patients who received cetuximab, univariate and multivariate analyses were performed to assess the effect of KRAS p.G13D mutations on progression-free survival (PFS) and overall survival (OS). Of the 98 patients, 23 (23.5\%) had KRAS p.G13D-mutated tumors, whereas 75 (76.5\%) had tumors harboring other mutations. Of the 31 patients who received cetuximab, 9 (29.0\%) had KRAS p.G13D mutations
\end{abstract}

Correspondence to: Dr Eiji Shinozaki, Department of Gastroenterology, The Cancer Institute Hospital, Japanese Foundation for Cancer Research, 3-8-31 Ariake, Koto-ku, Tokyo 135-8550, Japan E-mail: eiji.shinozaki@jfcr.or.jp

Key words: Kirsten rat sarcoma viral oncogene homolog p.G13D, cetuximab, vertical blockade of Ras pathway and $22(71.0 \%)$ had other mutations. There were no significant differences in age, gender, primary site, pathological type, history of chemotherapy, or the combined use of irinotecan between either of the patient subgroups. The univariate analysis revealed no significant difference in PFS or OS between the patients with KRAS p.G13D mutations and those with other mutations (median PFS, 4.5 vs. 2.8 months, respectively; $\mathrm{P}=0.65$; and median $\mathrm{OS}, 15.3$ vs. 8.9 months, respectively; $\mathrm{P}=0.51$ ). However, the multivariate analysis revealed a trend toward better PFS among patients harboring p.G13D mutations (PFS: HR=0.29; 95\% CI: 0.08-1.10; $\mathrm{P}=0.07$; OS: $\mathrm{HR}=0.23$; 95\% CI: 0.04-1.54; $\mathrm{P}=0.13$ ). In conclusion, treatment with cetuximab may be more clinically beneficial in $\mathrm{mCRC}$ patients with a KRAS p.G13D mutation, compared with those harboring other mutations. However, further investigation is required to clearly determine the benefits of cetuximab treatment in patients with KRAS p.G13D mutation-positive mCRC.

\section{Introduction}

The epidermal growth factor receptor (EGFR) is abundantly expressed in a broad spectrum of carcinomas, including colorectal adenocarcinoma.EGFR represents one of most promising molecules for targeted therapy of carcinomas. Cetuximab is a chimeric $\operatorname{Ig} \mathrm{G} 1$ monoclonal antibody that competitively binds to the EGFR extracellular domain with a higher affinity compared with endogenous ligands. Cetuximab mediates antibody-dependent cytotoxicity against human tumor cells. Several in vitro, in vivo and clinical studies have demonstrated that the efficacy of anti-EGFR monoclonal antibodies depends on the lack of v-Ki-ras2 Kirsten rat sarcoma viral oncogene homolog (KRAS) mutations. KRAS, a small G protein, is an essential component of the Ras/Raf/mitogen-activated protein kinase pathway, which may acquire oncogenic mutations that produce a constitutively active protein. Approximately $30-50 \%$ of patients with metastatic colorectal cancer (mCRC) 
have a KRAS mutation, which is a major predictive biomarker of resistance to anti-EGFR therapies. The most frequent mutations occur in codons 12 (G12D, 13\%; and G12V, 9\%) or 13 (G13D, 8\%) (1). Previous studies have suggested that patients who have tumors with KRAS G13D mutations are likely to respond better to cetuximab compared with those who have other KRAS mutations (2). In vitro as well as in vivo treatment with cetuximab significantly inhibited the growth of tumors formed by wild-type or KRAS p.G13D mutant cells (3). By contrast, the growth of tumors formed by KRAS p.G12V cells was not significantly affected by cetuximab treatment (3).

As regards the level of activation of EGFR and its downstream effectors, in the presence of cetuximab,p.G12V-mutated cells were seemingly still able to activate the extracellular signal-regulated kinase pathway, whereas p.G13D-mutated cells were not. Of note, the levels of activated KRAS were similar in p.G12V- and p.G13D-mutated cells. Overall, these results indicate that the KRAS p.G12V and p.G13D mutations differently affect the response to cetuximab in preclinical models (3). Similarly, KRAS codon 12 was reported to be a poor-prognosis gene in the PETTAC8 clinical trial, whereas codon 13 is apparently associated with a less poor prognosis (4). Anti-EGFR inhibitors may thus constitute new treatment options for patients with tumors harboring KRAS G13D mutations. The purpose of this retrospective analysis was to assess the prevalence of KRAS p.G13D mutations and evaluate the effectiveness of treatment with cetuximab in Japanese mCRC patients who harbor KRAS p.G13D or other KRAS mutations.

\section{Patients and methods}

Patients. The clinical records of $98 \mathrm{mCRC}$ patients who had genotyped KRAS mutations and were treated between August, 2004 and January, 2011 in four hospitals located in Tokyo and Kyushu Island, were reviewed to study the subtypes of KRAS mutations and patient characteristics. Of the 98 patients, 31 received regimens combining cetuximab with irinotecan, and their KRAS mutation status was evaluated by Luminex assays. In the combined regimen, cetuximab was administered at an initial dose of $400 \mathrm{mg} / \mathrm{m}^{2}$ followed by weekly intravenous infusions of $250 \mathrm{mg} / \mathrm{m}^{2}$, whereas irinotecan was administered once every 2 weeks as an intravenous infusion of $150 \mathrm{mg} / \mathrm{m}^{2}$, in accordance with the package insert for irinotecan in Japan. One treatment cycle in our study comprised cetuximab administered twice weekly plus irinotecan administered once every 2 weeks by intravenous infusion. Patients who met all of the following inclusion criteria were retrospectively included in analyses: i) histologically confirmed diagnosis of adenocarcinoma of the colon or rectum with evaluated KRAS status and ii) presence of unresectable metastatic disease.

This study was conducted following approval of the Science Review Board of the Cancer Institute Hospital (Tokyo, Japan).

KRAS testing. KRAS status was evaluated by Luminex assays. KRAS testing by Luminex assay and direct sequencing was performed by clinical testing companies (MBL, Nagoya, Japan; and SRL, Tokyo, Japan). The sensitivity of KRAS testing by Luminex has been reported to be $10 \%$ (5).
Statistical analysis. Progression-free survival (PFS) was defined as the time from the first day of treatment with cetuximab plus irinotecan, to either the first objective evidence of disease progression or death from any cause. Overall survival (OS) was defined as the time from the first day of treatment with cetuximab plus irinotecan to death from any cause. The principal investigators at our institution re-evaluated PFS and OS for all the patients. Fisher's exact tests were used to compare patient characteristics. OS and PFS were estimated using the Kaplan-Meier method, and differences among groups categorized according to KRAS status were compared using the log-rank test. All the reported P-values were derived by two-sided tests, with $\mathrm{P}<0.05$ considered to indicate statistically significant differences. The prognostic factors included age ( $<65$ or $\geq 65$ years), gender (male or female), performance status (PS; 0 or 1), primary site (colon, rectum and multiple), pathology (well- or moderately vs. poorly differentiated), and KRAS mutations (G13D or others). Prognostic factors with $\mathrm{P}<0.2$ on univariate analysis were included in multivariate analysis. The statistical analyses were performed using IBM SPSS statistics 18 software (SPSS Inc., Tokyo, Japan).

\section{Results}

Patient characteristics. Between September, 2008 and April, 2010, 98 patients met the inclusion criteria and were included in the analysis. Of the 98 patients, 23 (23.5\%) had tumors with KRAS p.G13D mutations, whereas the remaining $75(76.5 \%)$ had tumors with other mutations. Of the 98 patients, 31 received cetuximab: 9 (29.0\%) had KRAS p.G13D mutations and the remaining $22(71.0 \%)$ patients harbored other mutations. The frequency of tumors with KRAS p.13D mutations tended to be higher in female patients and in the colon. There were no significant differences in age, gender, primary site, pathological type, history of chemotherapy, or the combined use of irinotecan between either of these subgroups of patients. The characteristics of the patients are summarized in Table I.

Frequency of p.G13D mutations. A total of 24 patients had KRAS codon 13 mutations; 23 of these patients $(95.8 \%)$ had a KRAS p.G13D mutation, and only 1 patient (4.2\%) had a KRAS p.G13C mutation (Table II).

Between-study comparison of mutation frequency. The prevalence of KRAS p.G13D mutations was $23.5 \%$ in this study. There were no significant differences compared with the results of previous studies (Table III).

Characteristics of patients administered cetuximab as first-line treatment. Of the 31 patients who received cetuximab as first-line treatment, 9 (29\%) had a KRAS p.G13D mutation and $22(71 \%)$ had another type of KRAS mutation. There were no significant differences in the baseline clinical characteristics between these two groups (Table IV).

Overall survival (OS) and progression-free survival (PFS) according to the presence of p.G13D vs. other mutations. The median OS was 15.3 months in patients with KRAS p.G13D mutations ( $\mathrm{n}=9)$, compared with 8.9 months in those with other KRAS mutations $(n=22)(P=0.51$; Fig. 1$)$. The median PFS was 
Table I. Patient characteristics.

\begin{tabular}{|c|c|c|c|}
\hline Characteristics & $\begin{array}{c}\text { p.G13D mutation, no. }(\%) \\
n=23(23.5)\end{array}$ & $\begin{array}{l}\text { Other mutations, no. }(\%) \\
\qquad \mathrm{n}=75(76.5)\end{array}$ & P-value \\
\hline Age (years) & & & 0.14 \\
\hline Median (range) & $64.5(44.0-76.0)$ & $60.0(39.0-81.0)$ & \\
\hline$<65$ & $11(47.8)$ & $50(66.7)$ & \\
\hline$\geq 65$ & $12(52.2)$ & $25(33.3)$ & \\
\hline Gender & & & 0.05 \\
\hline Male & $8(34.8)$ & $45(60.0)$ & \\
\hline Female & $15(65.2)$ & $30(40.0)$ & \\
\hline Primary site & & & 0.08 \\
\hline Colon & 17 (73.9) & $39(52.0)$ & \\
\hline Rectum/anus & $5(21.7)$ & $34(45.3)$ & \\
\hline Multiple & $1(4.4)$ & $2(2.7)$ & \\
\hline Differentiation & & & 0.97 \\
\hline High & $8(34.8)$ & $22(29.3)$ & \\
\hline Moderate & $13(56.6)$ & $43(57.3)$ & \\
\hline Poor & $1(4.3)$ & $5(6.7)$ & \\
\hline Unknown & $1(4.3)$ & $5(6.7)$ & \\
\hline
\end{tabular}

Table II. KRAS mutations.

\begin{tabular}{ll}
\hline $\begin{array}{c}\text { Codon 12, no. (\%) } \\
\mathrm{n}=74(75.5)\end{array}$ & $\begin{array}{c}\text { Codon 13, no. }(\%) \\
\mathrm{n}=24(24.5)\end{array}$ \\
\hline G12D (GAT), $38(51.4)$ & G13D (GAC), $23(95.8)$ \\
G12S (AGT), 6 (8.1) & G13S (AGC), 0 (0.0) \\
G12C (TGT), 6 (8.1) & G13C (TGC), 1 (4.2) \\
G12R (CGT), 1 (1.3) & G13R (CGC), 0 (0.0) \\
G12V (GTT), 21 (28.4) & G13V (GTC), 0 (0.0) \\
G12A (GCT), 2 (2.7) & G13A (GCC), 0 (0.0) \\
\hline
\end{tabular}

KRAS, Kirsten rat sarcoma viral oncogene homolog.

4.5 months in patients with KRAS p.G13D mutations $(n=9)$, compared with 2.8 months in those with other KRAS mutations $(n=22)(P=0.65$; Fig. 2$)$.

Cox regression analysis of $O S$ and PFS. On the multivariate analysis, Eastern Cooperative Oncology Group PS was found to be independently associated with prolongation of OS and PFS. In addition, OS and PFS tended to be prolonged in patients with KRAS p.G13D mutations. However, the difference between patients with KRAS p.G13D mutations and those with other genotypes did not reach statistical significance (OS, $\mathrm{P}=0.13$; and PFS, $\mathrm{P}=0.07$ ) (Table V).

\section{Discussion}

Anti-EGFR therapy is currently approved for use only in patients with wild-type mCRC tumors. OS is $>30$ months in patients with KRAS wild-type mCRC, as compared with

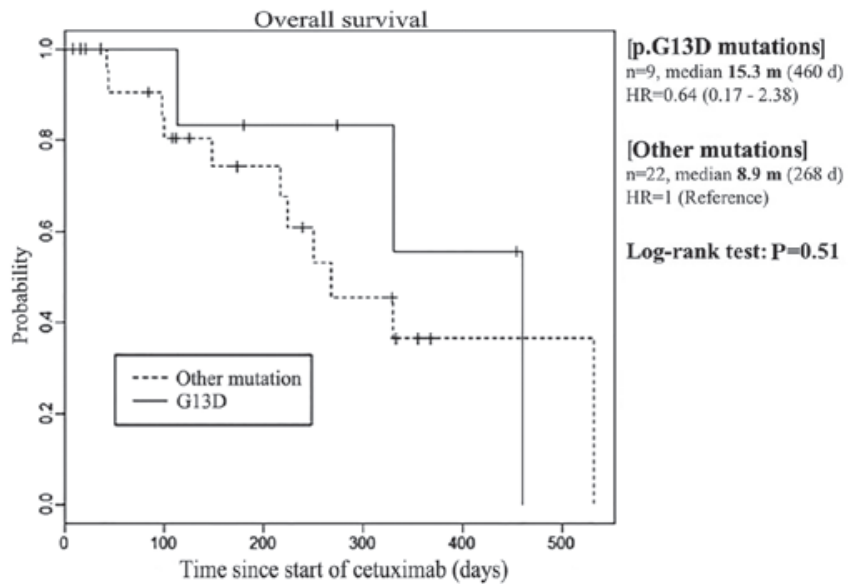

Figure 1. Overall survival curves according to KRAS p.G13D mutation or others $(n=31)$. HR, hazard ratio.

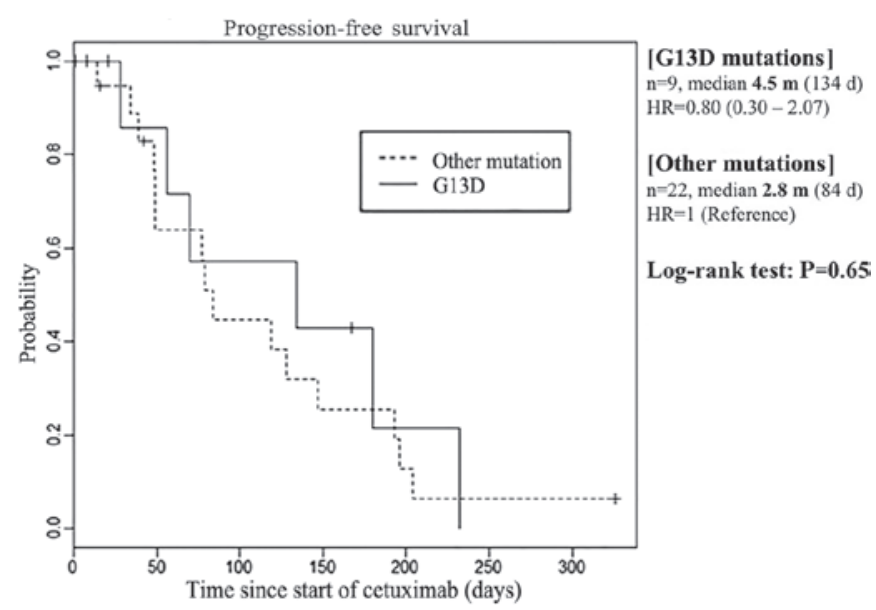

Figure 2. Progression-free survival curves according to KRASp.G13D mutation or others $(n=31)$. HR, hazard ratio. 
Table III. Comparison with previous studies.

\begin{tabular}{lcccc}
\hline Mutation type & $\begin{array}{c}\text { NCI CTG/AGITG } \\
(\mathrm{n}=164)\end{array}$ & $\begin{array}{c}\text { Leuven }^{\mathrm{b}} \\
(\mathrm{n}=122)\end{array}$ & $\begin{array}{c}\text { Italian }^{\mathrm{c}} \\
(\mathrm{n}=24)\end{array}$ & $\begin{array}{c}\text { This study (Japanese) } \\
(\mathrm{n}=98)\end{array}$ \\
\hline $\begin{array}{l}\text { p.G13D } \\
\text { Other }\end{array}$ & $20(12.2)$ & $20(16.4)$ & $5(20.8)$ & $23(23.5)$ \\
\hline
\end{tabular}

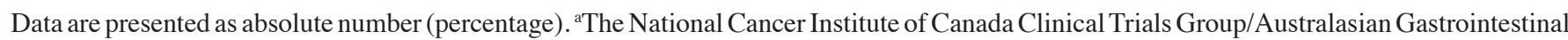
Trials Group. 'Leuven data set comprises EVEREST, BOND, SALVAGE and BABEL trial data. 'Italian data set comprises BOND, MABEL and EMR202600 trial data. The data are available at http://jama.ama-assn.org/content/304/16/1812/suppl/DC1.

Table IV. Patient characteristics.

\begin{tabular}{lcc}
\hline & $\begin{array}{c}\text { p.G13D mutations, no. }(\%) \\
\mathrm{n}=9(29.0)\end{array}$ & $\begin{array}{c}\text { Other mutations, no. }(\%) \\
\mathrm{n}=22(71.0)\end{array}$ \\
\hline Age (years) & & \\
Median (range) & $61.0(44.0-72.0)$ & $64.0(50.0-79.0)$ \\
$<65$ & $5(55.6)$ & $13(59.1)$ \\
$\geq 65$ & $4(44.4)$ & $9(40.9)$ \\
Gender & & $12(54.5)$ \\
Male & $3(33.3)$ & $10(45.5)$ \\
Female & $6(66.7)$ & $17(77.2)$ \\
ECOG PS & & $5(22.8)$ \\
0 & $6(66.7)$ & 0.43 \\
1 & $3(33.3)$ & $0.0)$ \\
2 & $0(0.0)$ & 0.65 \\
\hline
\end{tabular}

ECOG PS, Eastern Cooperative Oncology Group performance status.

Table V. Murtivariate analysis.

\begin{tabular}{|c|c|c|c|c|}
\hline & HR & Lower $95 \% \mathrm{CI}$ & Upper $95 \%$ CI & P-value \\
\hline \multicolumn{5}{|l|}{ Overall survival } \\
\hline Age, years $(<65$ vs. $\geq 65)$ & 1.86 & 0.50 & 6.84 & 0.35 \\
\hline Gender (M vs. F) & 1.50 & 0.39 & 5.78 & 0.55 \\
\hline ECOG-PS (0 vs. 1) & 5.29 & 1.39 & 20.2 & $<0.01$ \\
\hline KRAS (other vs. G13D) & 0.23 & 0.04 & 1.54 & 0.13 \\
\hline \multicolumn{5}{|l|}{ Progression-free survival } \\
\hline Age, years $(<65$ vs. $\geq 65)$ & 1.05 & 0.41 & 2.72 & 0.91 \\
\hline Gender (M vs. F) & 1.28 & 0.42 & 3.96 & 0.66 \\
\hline ECOG-PS (0 vs. 1) & 5.41 & 1.71 & 17.2 & $<0.01$ \\
\hline KRAS (other vs. G13D) & 0.29 & 0.08 & 1.10 & 0.07 \\
\hline
\end{tabular}

M, male; F, female; HR, hazard ratio; CI, confidence interval; KRAS, Kirsten rat sarcoma viral oncogene homolog; ECOG PS, Eastern Cooperative Oncology Group performance status.

only 20.3 months in patients with mCRC harboring KRAS exon 2 mutations (6). The results of further clinical trials of TAS-102 (7) or other new drugs for the treatment of mCRC with KRAS mutations are therefore eagerly awaited.
In this study, KRAS p.G13D mutations accounted for $23.5 \%$ of all KRAS mutations, which is consistent with the results of previous studies. However, the prevalence of KRAS p.G13D mutations was higher in female patients, which is not 
similar to the results of studies performed by another Japanese group (8) and other groups in Europe $(1,3)$. In patients who received cetuximab, OS and PFS were marginally but not significantly longer in patients with KRAS p.G13D mutations compared with patients with other KRAS mutations.

A pooled exploratory analysis of the Cetuximab Combined with Irinotecan in First-Line Therapy for Metastatic Colorectal Cancer (CRYSTAL) study and the Oxaliplatin and Cetuximab in First-Line Treatment of Metastatic Colorectal Cancer (OPUS) study recently reported the effect of KRAS p.G13D mutations on outcome in patients with mCRC who received first-line chemotherapy with or without cetuximab (1). Patients with p.G13D-mutant tumors appeared to benefit from first-line treatment with cetuximab, although there were no significant differences. On the other hand, in a subanalysis of the Medical Research Council COIN trial, a phase III trial evaluating the benefits of adding cetuximab to oxaliplatin-based first-line combination chemotherapy, in the PRIME study and studies 20050181 and 20020408, which were phase III trials evaluating the effectiveness of adding panitumumab to combination chemotherapy, patients with p.G13D-mutant tumors did not appear to benefit from first-line treatment with an anti-EGFR inhibitor (9).

A prospective phase II trial titled 'A Trial of Cetuximab with or without Irinotecan for Advanced Bowel Cancer (ICE CREAM)' is currently ongoing in the United Kingdom. The aim of this trial is to validate that cetuximab alone or combined with irinotecan is effective for advanced bowel cancer with p.G13D mutations, and the results are eagerly awaited. However, it remains unclear whether we should use an anti-EGFR inhibitor to treat patients who have mCRC with KRAS p.G13D mutations. Therefore, we do not recommend an anti-EGFR inhibitor for such patients at present.

An important topic for future research is vertical blockade of the Ras pathway. Previous in vitro studies reported that, given the higher efficacy of triple-drug therapy [a mitogen-activated protein kinase kinase (MEK) and phosphoinositide 3-kinase (PI3K)/mammalian target of rapamycin (mTOR) inhibitor combined with cetuximab], periodic administration of anti-EGFR antibodies may more effectively delay tumor progression (10). Concomitant blockade of MEK and PI3K/mTOR induced high rates of disease stabilization in patient-derived $\mathrm{mCRC}$ xenografts harboring KRAS, neuroblastoma RAS viral oncogene homolog (NRAS), BRAF and/or PIK3 catalytic subunit $\alpha$ mutations. These findings suggest potential therapeutic opportunities to delay disease progression by several alternative treatment options in patients who have tumors with KRAS or other mutations (10).

The present study had several limitations. Most importantly, it was a retrospective study and the sample size was small. In addition, we did not evaluate other biomarkers, such as KRAS exon 3 or 4, NRAS, or BRAF mutations. Although cetuximab may be more clinically beneficial for $\mathrm{mCRC}$ patients with KRAS p.G13D mutations compared with those harboring other mutations, further studies are required to elucidate the potential benefits of cetuximab treatment for $\mathrm{mCRC}$ patients with KRAS p.G13D mutations.

In conclusion, the use of an anti-EGFR antibody is not currently recommended for patients with mCRC associated with KRAS p.G13D mutations; however, as for the combination with a drug blocking the tumor growth pathway perpendicularly, it it likely to be effective.

\section{References}

1. Tejpar S, Celik I, Schlichting M, Sartorius U, Bokemeyer C and Van Cutsem E: Association of KRAS G13D tumor mutations with outcome in patients with metastatic colorectal cancer treated with first-line chemotherapy with or without cetuximab. J Clin Oncol 30: 3570-3577, 2012.

2. Mao C, Huang YF, Yang ZY, Zheng DY, Chen JZ and Tang JL: KRAS p.G13D mutation and codon 12 mutations are not created equal in predicting clinical outcomes of cetuximab in metastatic colorectal cancer: a systematic review and meta-analysis. Cancer 119: 714-721, 2013.

3. De Roock W, Jonker DJ, Di Nicolantonio F, Sartore-Bianchi A Tu D, Siena S, Lamba S, Arena S, Frattini M, Piessevaux H, et al: Association of KRAS p.G13D mutation with outcome in patients with chemotherapy-refractory metastatic colorectal cancer treated with cetuximab. JAMA 304: 1812-1820, 2010.

4. Blons H, Emile JF, Le Malicot K, Julié C, Zaanan A, Tabernero J, Mini E, Folprecht G, Van Laethem JL, Thaler J, et al: Prognostic value of KRAS mutations in stage III colon cancer: Post hoc analysis of the PETTAC8 phase III trial dataset. Ann Oncol 25: 2378-2385, 2014.

5. Fukushima Y, Yanaka S, Murakami K, Abe Y, Koshizaka T, Hara H, Samejima C, Kishi Y, Kaneda M and Yoshino T: High-throughput screening method of KRAS mutations at codons 12 and 13 in formalin-fixed paraffin-embedded tissue specimens of metastatic colorectal cancer. Cancer \& chemotherapy 38: 1825-1835, 2011 (In Japanese).

6. Heinemann V, von Weikersthal LF, Decker T, Kiani A, Vehling-Kaiser U, Al-Batran SE, Heintges T, Lerchenmüller C, Kahl C, Seipelt G, et al: FOLFIRI plus cetuximab versus FOLFIRI plus bevacizumab as first-line treatment for patients with metastatic colorectal cancer (FIRE-3): A randomised, open-label, phase 3 trial. Lancet Oncol 15: 1065-1075, 2014.

7. Yoshino T, Mizunuma N, Yamazaki K, Nishina T, Komatsu Y, Baba H, Tsuji A, Yamaguchi K, Muro K, Sugimoto N, et al: TAS-102 monotherapy for pretreated metastatic colorectal cancer: A double-blind, randomised, placebo-controlled phase 2 trial. Lancet Oncol 13: 993-1001, 2012.

8. Bando H, Yoshino T, Yuki S, Shinozaki E, Nishina T, Kadowaki S, Yamazaki K, Kajiura S, Tsuchihara K, Fujii S, et al: Clinical outcome of Japanese metastatic colorectal cancer patients harbouring the KRAS p.G13D mutation treated with cetuximab + irinotecan. Jpn J Clin Oncol 42: 1146-1151, 2012.

9. Peeters M, Douillard JY, Van Cutsem E, Siena S, Zhang K, Williams R and Wiezorek J: Mutant KRAS codon 12 and 13 alleles in patients with metastatic colorectal cancer: Assessment as prognostic and predictive biomarkers of response to panitumumab. J Clin Oncol 31: 759-765, 2013.

10. Migliardi G, Sassi F, Torti D, Galimi F, Zanella ER, Buscarino M, Ribero D, Muratore A, Massucco P, Pisacane A, et al: Inhibition of MEK and PI3K/mTOR suppresses tumor growth but does not cause tumor regression in patient-derived xenografts of RAS-mutant colorectal carcinomas. Clin Cancer Res 18: 2515-2525, 2012. 